\title{
THE INFLUENCE OF QUERCETIN ON THE INTERACTION OF HALOPERIDOL WITH HUMAN SERUM ALBUMIN
}

Miroslav Sovrlić ${ }^{1}$, Aleksandar Kočović ${ }^{1}$, Ratomir Jelić ${ }^{1}$, Jovica Tomovic ${ }^{1}$, Anđela Uštević ${ }^{1}$, Emina Mrkalić ${ }^{2}$

${ }^{1}$ Faculty of Medical Sciences, Department of Pharmacy, University of Kragujevac, 34000 Kragujevac, Serbia

${ }^{2}$ Institute for Information Technologies Kragujevac, Department of Science, 34000 Kragujevac, Serbia

Corresponding author: Aleksandar Kočović; e-mail: salekkg91@gmail.com

\section{INTRODUCTION}

Protein-binding interactions are displacement reactions which have been implicated as the causative mechanisms in many drugdrug, food-drug and plant-drug interactions. Since only the free fraction of the drug is active in the body, it is clear that changes in the binding of the drug to plasma proteins can be of great importance for the effect of the drug as well as for the occurrence of side effects. Thus, the aim of presented study was to analyze human serum albumin-binding displacement interaction between two ligands, haloperidol (HPD) and widely distributed plant flavonoid quercetin.

\section{METHODS}

Fluorescence analysis was used in order to investigate the effect of substances on intrinsic fluorescence of human serum albumin (HSA) and to define binding and quenching properties of ligandalbumin complexes in binary and ternary systems, respectively. All measurments were done on RF-1501 PC spectrofluorometer (Shimadzu, Japan) on two temperatures (303 K and $310 \mathrm{~K}$ ). For data analysis Stern-Volmer eqution was used. Both ligands showed the ability to bind to HSA, although to a different extent. The displacement effect of one ligand from HSA by the other one has been described on the basis of the quenching curves and binding constants comparison for the binary and ternary systems.

\begin{tabular}{|c|c|c|c|c|}
\hline $\begin{array}{c}\text { Temperature } \\
\text { (K) }\end{array}$ & Ksv (mol/L) & $\mathrm{Ka}(\mathrm{mol} / \mathrm{L})$ & $\mathrm{n}$ & $\mathrm{R}^{2}$ \\
\hline 303 & $3.5 \times 10^{3}$ & $4.07 \times 10^{3}$ & 1.02 & 0.9920 \\
\hline 310 & $3.61 \times 10^{3}$ & $1.95 \times 10^{3}$ & 0.93 & 0.9900 \\
\hline
\end{tabular}

\section{RESULTS}

Fluorescent spectroscopic data showed that the fluorescence quenching of HSA results from the formation of the HPD -HSAquercetin complex. Spectroscopic analyses at different temperatures indicate that the mechanism of extinguishing the human serum albumin and the quercetin dynamic process. Process constants ( $K a$ and Ksv) and connective sites ( $n$ ), of interaction between HPD and HAS at $303 \mathrm{~K}$ and $310 \mathrm{~K}$ are given in Table 1. Process constants (Ka and Ksv) and connective sites (n), of interaction between HPD, HSA and quercetin at $303 \mathrm{~K}$ and $310 \mathrm{~K}$ are given in Table 2.

Table 2: Process constants ( $K a$ and Ksv) and connective sites ( $n$ ), of interaction between HPD, HSA and quercetin at different

temperatures

\begin{tabular}{|c|c|c|c|c|}
\hline $\begin{array}{c}\text { Temperature } \\
(\mathrm{K})\end{array}$ & $\mathrm{Ksv}(\mathrm{mol} / \mathrm{L})$ & $\mathrm{Ka}(\mathrm{mol} / \mathrm{L})$ & $\mathrm{n}$ & $\mathrm{R}^{2}$ \\
\hline 303 & $9.65 \times 10^{2}$ & $3.75 \times 10^{2}$ & 0.89 & 0.9833 \\
\hline 310 & $9.19 \times 10^{2}$ & $4.28 \times 10^{2}$ & 0.92 & 0.9573 \\
\hline
\end{tabular}

\section{CONCLUSION}

It was shown that HPD and quercetin compete for the same binding site (Sudlow's site I) on HSA molecule. They competitive binding leads to decrease in the investigated constants indicating that the binding of quercetin to HSA leads to a lower binding of haloperidol to HSA, which can potentially result in an increase in the free fraction of the drug in the plasma and the occurrence of adverse effects in patients on treatment with HPD.

References:

1. Berić JD, Stojanović SD, Mrkalić EM, Matović ZD, Milovanović DR, Sovrlić MM, Jelić RM. Interaction of haloperidol with human serum albumin and effect of metal ions on the binding. Monatshefte für Chemie-Chemical Monthly. 2018;149(12):2359-68.

2. Kameníková M, Furtmüller PG, Klacsová M, Lopez-Guzman A, Toca-Herrera JL, Vitkovská A, Devínsky F, Mučaji P, Nagy M. Influence of quercetin on the interaction of gliclazide with human serum albumin-spectroscopic and docking approaches. Luminescence. 2017;32(7):1203-11. 